\title{
Research on VM Dynamic Scheduling in Cloud Environment
}

\author{
Hanqian $\mathrm{Wu}^{1,2, \mathrm{a}}$, Tao $\mathrm{Chen}^{1,2, \mathrm{~b}}$, Jue $\mathrm{Xie}^{3, \mathrm{c}}$ \\ ${ }^{1}$ School of Computer Science and Engineering, Southeast University, Nanjing 210018, China \\ ${ }^{2}$ Key Laboratory of Computer Network and Information Integration of Ministry of Education, \\ Southeast University, Nanjing 210018, China \\ ${ }^{3}$ School of Information Technology Southeast University-Monash University Joint Graduate School \\ Suzhou, China \\ ahanqian@seu.edu.cn,'chentao_ct@outlook.com, cjue.xie@monash.edu
}

Keywords: cloud computing,VM dynamic Scheduling, multi-objective, BFD, ARIMA.

Abstract. In order to improve the uneven utilization of resources, electricity power loss and QoS (Quality of Service) in the cloud environment, a multi-objective VM (virtual machine) dynamic Scheduling mechanism is proposed. The VM in the cloud environment is dynamically managed via designing the strategy of migration triggering, selection of migrating VM and dynamic placement of VM. In the proposed mechanism, the model of ARIMA is employed to predict the utilization rate of physical host resources. Moreover, the improved BFD algorithm plays the role of allocating VMs dynamically according to the dual-threshold trigger migration. The empirical results demonstrate that the load balancing of physical hosts can be effectively ensured and the power consumption can be reduced while maintaining a slow SLA violation rate.

\section{Introduction}

One of the areas of greatest interest in recent years is cloud computing, which has played an important role in the current IT industry[1]. Now, including Amazon, Microsoft, Google, Alibaba, Tencent, Huawei and other companies have established public cloud services. There are some other Internet companies also have their own private cloud[2]. The management of VM has been a hot issue in the industrial and academic research[3].

The current goals of optimizing VM management are SLA, power loss and resource balance of this several aspects. In response to the corresponding goals, some strategies for dynamic scheduling of VMs have been proposed. Some researchers save money by saving energy consumption, according to SLA on the revenue, while maintaining the SLA violation rate in a particular section which aims to maximize cost savings. According to the pre-set threshold, a set of energy migration-oriented VM migration mechanism was designed to predict the utilization of physical host resources so as to trigger migration, which employs the relationship between CPU utilization and power consumption and AR (Autoregressive Model)[4].Skewnesscan be used to measure the uneven utilization of multiple resources of each VM and according skew value load balance across VMs[5].

Traditional VM dynamic management strategy optimization goals are mainly SLA, power loss and resource balance but it is not common to combine the three goals together. At present, the dual-threshold policy is mainly used for triggering the migration of VMs. The upper and lower thresholds for triggering migration are set according to specific resources, but the instability of resource utilization is seldom considered.In this paper, the dynamic scheduling strategy of VMs is proposed to consider multiple objectives synthetically. ARIMA time series prediction model is used 
to predict the utilization of host resources and eliminate the adverse effects of instability of resource utilization. Finally,improved traditional heuristic algorithm is utilized to dynamically place the VM while balancing the conflict between targets.

VM dynamic scheduling mechanism. The process of VM dynamic scheduling involves the VM and the physical machine rescheduling or matching, so as to meet the changing demands of VM resource and provide scalability and reliability. The causes of these situations are the termination of the application, the large number of user requests, the user suspending the executing program, or the different application resource requirements over time.VM dynamic scheduling is which VM selected and placed on their physical machine.It is mainly divided into three parts, including migration triggering strategy, migration of VM selection strategy and placement of host selection strategy.

Model definition. The objectives considered in this paper mainly include SLA, power loss and resource balance.

1) SLA model:The service level agreement is part of the contract between the customer and the service provider.SLA is generally used to define the level of service. We can express the SLA violation rate based on the amount of resources requested by $\mathrm{VM} v_{i}$ and the amount of resources allocated to the VM by the physical host. $r_{i}^{c p u}, r_{i}^{m e m}$ and $r_{i}^{\text {net }}$ represent the size of the VM $v_{i}$ request CPU, memory, and bandwidth respectively. $a_{i}^{c p u}, a_{i}^{m e m}$ and $a_{i}^{\text {net }}$ represent the size of CPU, memory and bandwidth allocated by the physical host to the VM $v_{i}$ respectively. The SLA evaluation function is expressed as:

$$
S_{j}^{c p u}=\frac{\sum_{i \in V}\left(r_{i}^{c p u}-a_{i}^{c p u}\right)}{\sum_{i \in V} r_{i}^{c p u}}, S_{j}^{m e m}=\frac{\sum_{i \in V}\left(r_{i}^{m e m}-a_{i}^{m e m}\right)}{\sum_{i \in V} r_{i}^{m e m}}, S_{j}^{n e t}=\frac{\sum_{i \in V}\left(r_{i}^{\text {net }}-a_{i}^{\text {net }}\right)}{\sum_{i \in V} r_{i}^{\text {net }}}, S_{j}=\frac{S_{j}^{c p u}+S_{j}^{m e m}+S_{j}^{n e t}}{3}
$$

The range of $S$ is $[0,1]$, and the larger the value is, the higher the SLA violation rate will be.

2)Resource load balance:Because each VM has different requirements for each resource of the physical nodes, resulting in different changes of the remaining resources on each physical node. In order to avoid occasions that the quantity of some resources is overabundant or barely sufficient,we need to try to maintain the balance among the various resources of each physical node.The resources requested by the VM are mainly CPU, memory and bandwidth. We use $\bar{u}_{j}$ represents the mean value of the three kinds of resources.

$$
L_{j}=\frac{3}{\left(\frac{u_{j}^{c p u}}{\bar{u}_{j}}\right)^{2}+\left(\frac{u_{j}^{m e m}}{\bar{u}_{j}}\right)^{2}+\left(\frac{u_{j}^{\text {net }}}{\bar{u}_{j}}\right)^{2}}(5)
$$

3) Power loss. When a physical host is running, the host's power consumption is related to his load factor. Memory, bandwidth and hard disk have little impact on the power consumption[5].To 
some extent, other factors can be ignored. So the power consumption is associated with linear CPU utilization. Then, considering the power consumption of a host, we mainly consider its power consumption in idle time and based on CPU utilization to calculate the host's power consumption in non-idle time. The power consumption of host $h_{j}$ is defined as follows:

$$
P_{j}=P_{j}^{\text {idle }}+\left(P_{j}^{\text {busy }}-P_{j}^{\text {idle }}\right) \times u_{j}^{C P U}
$$

$P_{j}^{i d l e}$ and $P_{j}^{\text {busy }}$ respectively represent the power loss of the physical host $h_{j}$ when it is fully loaded and unloaded. $u_{j}^{C P U}$ represents the utilization of the host $h_{j}$ of CPU.

Migration trigger strategy.The conditions for migration need to be set using the state of the running node, mainly collecting CPU, hard disk, memory, and bandwidth usage.Low load and overload are the two states of the host in both cases, the VM migration on the host should be triggered to ensure that the host is load balanced.Therefore, monitoring the resource utilization status of all active hosts in the cloud environment in real time is the primary task of the VM trigger.However, the utilization rate of the host resource is unstable.In this case, it is inaccurate to decide whether or not to migrate the VM according to the current resource utilization. So we use ARIMA time series forecasting model to predict physical host resource utilization rate, which is more universal.

We set dual-threshold to trigger the migration which is $\left(R_{\min }, R_{\max }\right), R_{\max }$ is used to indicate the upper limit of the current resource usage which is mainly used to prevent the current resources from being insufficient which leads to the decrease of system performance and affects the user experience. $R_{\min }$ is used to indicate the lower limit of resource usage which is mainly used to prevent the idle host from consuming the power so as to save energy.

In this paper, we use the ARIMA model to analyze the data and set it to $R$.According to the double-threshold set in this paper, $R$ is compared with double threshold $\left(R_{\min }, R_{\max }\right)$. When each value in $R$ is less than the value of $R_{\min }$ (indicated by $R<R_{\min }$ ), a low-load migration trigger algorithm is used.All active state VMs on the physical host are migrated to other physical hosts, and the physical host is hibernated to save power consumption. When each value in $R$ is greater than the value of $R_{\max }$ (indicated by $R>R_{\max }$ ), an overload migration trigger algorithm is used.

VM Selection Strategy.In the case of overload triggers we need to further determine which ones are to be migrated to the target VM. This article makes further judgment based on memory resources.

$$
\text { Ratio }_{i}=R_{i}^{\text {cpu }} / R_{i}^{\text {mem }} \quad, \quad \quad \text { Multip }_{i}=R_{i}^{\text {cpu } *} R_{i}^{\text {mem }}
$$

In the expression, $R_{i}^{c p u}$ represents the utilization of VM $v_{i}$ CPU. $R_{i}^{\text {mem }}$ represents the utilization of 
VM $v_{i}$ memory. We set a maximum memory usage for the physical host threshold $M E N_{\max }$, the value of $90 \%$. The threshold is mainly used when the memory usage of the host is too high, it can reduce the impact of high memory usage on the user experience by migrating more memory-intensive VMs. So when physical host $h_{j}$ memory utilization $M E N_{j}$ is greater than or equal to $M E N_{\max }$, we sort the VMs on the host in descending order according to the value of Multip $_{i}$, and then select the VMs to migrate them in sequence until $R_{j}<R_{\max }$. When $M E N_{j}$ is less than $M E N_{\max }$, The impact of the memory resource on the user experience is small. In this case, you should select VMs that use less memory and use more CPU resources. Since the host memory used by the VM is directly proportional to the cost of its migration (migration time), we need to take this factor into account at this time. We sort the VMs on host $h_{j}$ in descending order by Ratio $_{i}$ value and then migrate the VMs in order until $R_{j}<R_{\max }$.

Target host selection strategy.Currently, there are two main methods used.First, heuristic algorithms including first-time adaptive algorithm (FF), best-fit algorithm (BF) and descending best-fit algorithm (BFD). Second, evolutionary algorithms, including genetic algorithms and Ant colony algorithm. Although the evolutionary algorithm can calculate the better results, the calculation is complex, the number of iterations is large and slow convergence.Such algorithms are not suitable for the cloud environment which has more hosts.Therefore, this paper uses the traditional heuristic algorithm. We improve the BFD algorithm to complete the placement of VMs.

$D_{\text {min }}=\left(d_{\min }^{c p u}, d_{\min }^{\text {mem }}, d_{\min }^{\text {net }}\right)$ denotesthe minimum value of resources (CPU, memory, bandwidth) required for the physical host in the $\mathrm{VM}$ set $\mathrm{V}$ to be migrated. The pseudo code of the VM multi-objective dynamic placement algorithm is as follows:

Table 1 The pseudo code of the VM multi-objective dynamic placement algorithm

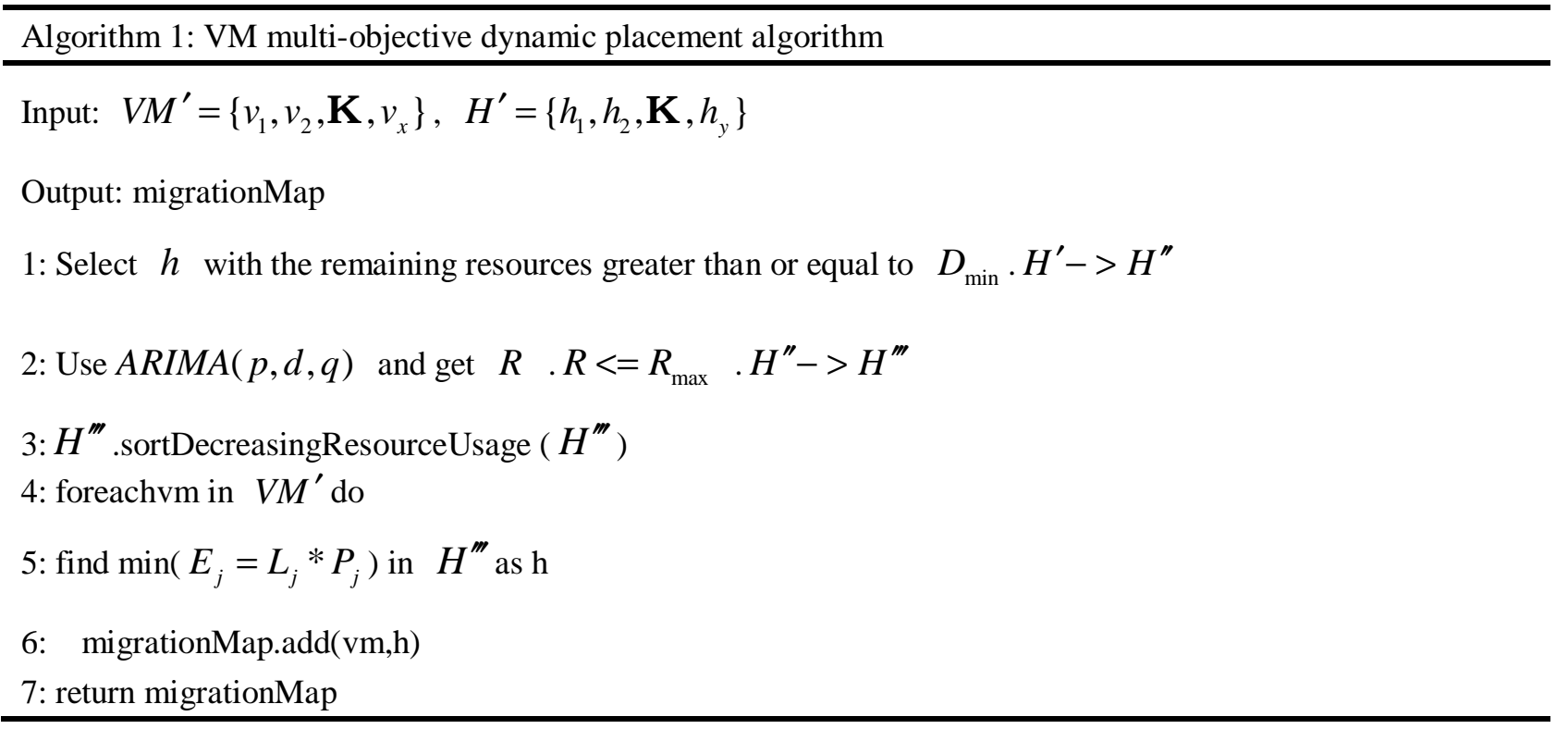

Experiment and result analysis. This paper uses CloudSim cloud simulation platform to simulate 
the cloud environment data center with hundreds of physical hosts to verify the validity of the proposed resource dynamic scheduling strategy.

On the platform, we create a data center consisting of 800 physical hosts, the configuration of which is based on HP host configuration. We divided the host processor capacity (MIPS) into two groups. Eachhost configuration is shown in Table 2.

Table 2 Simulated Data Center Physical Host Configuration

\begin{tabular}{llllll}
\hline No. & CPU & Core & Memory & bandwidth & Disk \\
\hline 1 & $1860 \mathrm{MHz}$ & 2 & $4 \mathrm{~Gb}$ & $1 \mathrm{~Gb} / \mathrm{s}$ & $1 \mathrm{~Gb}$ \\
2 & $2660 \mathrm{MHz}$ & 2 & $4 \mathrm{~Gb}$ & $1 \mathrm{~Gb} / \mathrm{s}$ & $1 \mathrm{~Gb}$ \\
\hline
\end{tabular}

In the data center composed of these nodes, 1050 virtual machines are created. The virtual machine MIPS is generated and distributed evenly from $\{2500,2000,1000,500\}$, respectively, with the memory being generated and evenly distributed from $\{870,1740,1740,613\}$, respectively, with a bandwidth of $100 \mathrm{Mbit} / \mathrm{s}$ and a disk size of $2.5 \mathrm{~Gb}$.We evaluated several common resource scheduling strategies, including non-target strategy and energy-saving dynamic voltage-frequency modulation (DVFS) strategy, single-threshold and dual-threshold dynamic scheduling strategy. We compared multi-objective resources dynamic scheduling proposed in the paper with those strategies.

Table 3 the power loss and SLA violation rate of each resource scheduling strategy

\begin{tabular}{lll}
\hline Strategy & power loss & SLA \\
\hline non-target & $2410.80 \mathrm{KWh}$ & - \\
DVFS & $803.91 \mathrm{KWh}$ & - \\
single-threshold & $191.73 \mathrm{KWh}$ & $10.14 \%$ \\
dual-threshold & $183.61 \mathrm{KWh}$ & $10.03 \%$ \\
multi-objective & $134.75 \mathrm{KWh}$ & $10.13 \%$ \\
\hline
\end{tabular}

From Table 2, we can see that the dynamic scheduling strategy can achieve energy-saving goals more than the static scheduling strategy. The dynamic scheduling strategy of this paper maintains a $29.7 \%$ and $26.6 \%$ reduction in energy consumption compared to the single-threshold and dual-threshold dynamic strategies, respectively, while maintaining a relatively low SLA violation rate.

We evaluated the resource load balancing for single-threshold, dual-threshold and multi-objective resource dynamic scheduling. We randomly selected a certain moment and analyzed current active physical host resources every 300 seconds. 


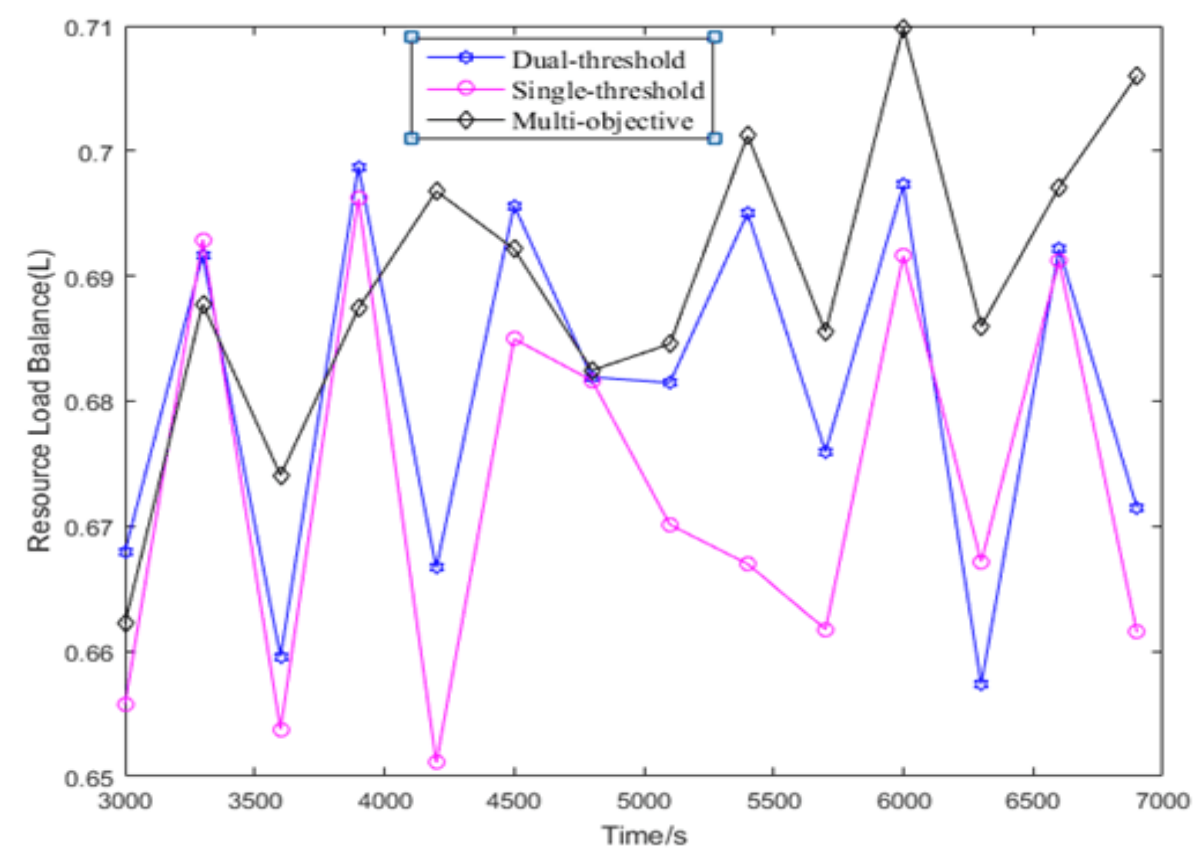

Fig. 1Three resource dynamic strategy resource load balancing

It can be seen from Fig. 1 that the resource balance of each host in the multi-objective resources dynamic scheduling strategy in this paper is slightly higher than that of single-threshold and double-threshold dynamic strategies.It shows that the resource utilization balance of the multi-objective resource dynamic scheduling strategy proposed is better in this paper.

\section{Conclusions}

Aiming at the problem of high power consumption and severe power waste in the cloud environment, this paper proposes a multi-objective resources dynamic scheduling strategy under the premise of fully considering SLA and resource load balancing. The strategy improves the balance of resource load and reduce power loss while ensuring that the SLA violation rate is relatively low.ARIMA model is used in the strategy to forecast the utilization of the host resources which can effectively avoid the instability of the utilization of the host resources. By improving the BFD packing algorithm the VM redistribution can ensure resource load balancing and SLA violation rate to a certain extent.

\section{References}

[1] G.B.Hima Bindu,Dr. J. Janet.A statistical survey on vm scheduling in cloud workstation for reducing energy consumption by balancing load in cloud [C]//2017 International Conference on Networks \& Advances in Computational Technologies (NetACT).Trivandrum, India,2017:34-43.

[2] Shi W, Hong B. Towards Profitable Virtual Machine Placement in the Data Center[C]// IEEE International Conference on Utility \& Cloud Computing. IEEE, 2011:138-145.

[3] Sakellariou R, Sakellariou R. Mapping Virtual Machines onto Physical Machines in Cloud Computing: A Survey[M]. ACM, 2016.

[4] Yang G, Zhang W. Research of optimized resource allocation strategy based on Openstack[C]// International Computer Conference on Wavelet Active Media Technology and Information Processing. IEEE, 2016:365-368. 
[5] Nagpure M B, Dahiwale P, Marbate P. An efficient dynamic resource allocation strategy for $\mathrm{VM}$ environment in cloud[C]// International Conference on Pervasive Computing. IEEE, 2015:1-5.

[6] Beloglazov A, Buyya R. Energy Efficient Resource Management in Virtualized Cloud Data Centers[C]// Ieee/acm International Conference on Cluster, Cloud and Grid Computing. IEEE Computer Society, 2010:826-831.

[7] Ismail H A, Riasetiawan M. CPU and memory performance analysis on dynamic and dedicated resource allocation using XenServer in Data Center environment[C]// International Conference on Science and Technology-Computer. IEEE, 2017:17-22.

[8] Zhang Q, Zhani M F, Boutaba R, et al. Dynamic Heterogeneity-Aware Resource Provisioning in the Cloud[J]. IEEE Transactions on Cloud Computing, 2014, 2(1):14-28.

[9] Bane R R, Annappa B, Shet K C. Survey of dynamic resource management approaches in virtualized data centers[C]// IEEE International Conference on Computational Intelligence and Computing Research. IEEE, 2014:1-7.

[10] Corradi A, Fanelli M, Foschini L. VM consolidation: A real case based on OpenStack Cloud[J]. Future Generation Computer Systems, 2014, 32(1):118-127. 